\section{Intersections}

Canadian Journal of Music

Revue canadienne de musique
Intersections CANADIAN JOURAL OF MUSIC
REVUE CANADIENEE DE MUSIOUH

\title{
Les chroniques musicales de Léo-Pol Morin - vecteur d'influence pour une réception québécoise de la modernité musicale française
}

\section{Paul Bazin}

Volume 32, numéro 1-2, 2012

URI : https://id.erudit.org/iderudit/1018578ar

DOI : https://doi.org/10.7202/1018578ar

Aller au sommaire du numéro

Éditeur(s)

Canadian University Music Society / Société de musique des universités canadiennes

ISSN

1911-0146 (imprimé)

1918-512X (numérique)

Découvrir la revue

Citer cet article

Bazin, P. (2012). Les chroniques musicales de Léo-Pol Morin — vecteur d'influence pour une réception québécoise de la modernité musicale française. Intersections, 32(1-2), 43-60. https://doi.org/10.7202/1018578ar
Résumé de l'article

Figure d'importance du milieu musical québécois au début du XX siècle,Léo-Pol Morin (1892-1941) a largement contribué à l’intégration du répertoire de la modernité surles scènes musicales du Canada français. Sa solide formation musicale, acquise autant au Québecqu'en France, ainsi que sa plume agile, ont fait de Morin un nom incontournable de la vie musicalemontréalaise. Interprète chevronné, ses idées et ses convictions sur la musique de son tempstrouvèrent une tribune de choix pendant plus de vingt ans dans des journaux tels que LaPatrie,La Presse et Le Canada. Cet article propose un survol des premières années de laparticipation de Léo-Pol Morin à la vie des concerts ainsi qu'une analyse de ses nombreux écritsportant sur les compositeurs de l'école moderne française, au coeur de laquelle il place GabrielFauré, Claude Debussy et Maurice Ravel.
Copyright @ C Canadian University Music Society / Société de musique des universités canadiennes, 2013
Ce document est protégé par la loi sur le droit d'auteur. L’utilisation des services d’Érudit (y compris la reproduction) est assujettie à sa politique d'utilisation que vous pouvez consulter en ligne.

https://apropos.erudit.org/fr/usagers/politique-dutilisation/ 


\title{
LES CHRONIQUES MUSICALES DE LÉO-POL MORIN - VECTEUR D'INFLUENCE POUR UNE RÉCEPTION QUÉBÉCOISE DE LA MODERNITÉ MUSICALE FRANÇAISE
}

\author{
Paul Bazin
}

Léo-Pol Morin (1892-1941) a consacré sa carrière à la défense de l'art musical au cœur de la province du Québec. L'influence de ce musicien polyvalent s'est fait sentir sur divers plans de cette scène musicale où il a agi à la fois à titre de critique et musicographe, de pianiste, de pédagogue et de compositeur. Riche d'une culture en partie acquise au cours de ses nombreux voyages en Europe et aux États-Unis, Léo-Pol Morin a revendiqué la rénovation en profondeur des mœurs musicales de l'époque en insistant sur l'idée qu'une réelle appréciation de l'art par le public doit reposer sur le goût de ses qualités intrinsèques, qu'autorise la compréhension élémentaire de son langage.

C'est en toute conscience des dispositions musicales des mélomanes auxquels il s'adresse qu'entre 1915 et 1941, Morin propose à ses lecteurs de nouvelles réflexions sur la nature des répertoires méconnus du nouveau $\mathrm{XX}^{\mathrm{e}}$ siècle, qu'il les instruit, notamment, par le biais d'une plume exigeante et éclairée, au sujet de cette musique qu'il considère pourtant être à portée de leur sensibilité. Le choix du répertoire sélectionné dans une telle entreprise pédagogique souligne d'ailleurs la conscience aigüe qu'il avait des dispositions des auditoires face à la nouvelle musique.

Les perspectives adoptées par Morin dans ses rubriques hebdomadaires à fort penchant musicographique mettent la musique de la modernité française à l'avant-plan de son discours ${ }^{1}$, celle-là même qu'il défend en tant que pianiste. Il se fait le porte-parole de ce répertoire, louant ses beautés en toute occasion, et s'est dévoué à en cultiver la connaissance et le goût auprès de ses compatriotes en consacrant régulièrement des textes à des compositeurs ciblés. Nombreux sont ceux qui bénéficièrent de son acharnement, de sa passion et de la force de ses convictions: il consacra des textes à des créateurs d'allégeances variées, tels que Gounod (1818-1893), Dukas (1865-1935), d'Indy (1851-1931) et les membres du Groupe des Six. Aussi cette multitude de compositeurs gravite-t-elle autour d'un groupe de trois noms revêtus d'une importance toute particulière au cœur

1 La littérature musicologique convient généralement de cet état de fait. Afin d'en faire définitivement la démonstration, il reste toutefois encore à produire un inventaire nominarum de tous ses écrits afin d'obtenir des statistiques pouvant faire la preuve concrète des compositeurs les plus cités. 
des écrits de Léo-Pol Morin, des trois piliers de sa pensée moderniste: Gabriel Fauré (1845-1924), Claude Debussy (1862-1918) et Maurice Ravel (1875-1937).

Les stratégies et les motivations latentes adoptées par Morin dans la promotion de la musique de ces compositeurs apparaissent au terme de la lecture attentive d'une sélection de textes traitant soit de compositeurs précis, soit de l'état de l'auditoire de son temps et ainsi, par extension, du rôle du critique musical. L'analyse d'un tel discours éclaire le chercheur quant au caractère pédagogique sous-jacent aux écrits de Morin dans la presse généraliste québécoise, et permet d'évaluer l'élargissement potentiel de ce que Hans Robert $\mathrm{Jau} \beta^{2}$ nomme l'horizon d'attentes, sous l'influence du discours engagé de LéoPol Morin.

\section{LÉo-Pol MORIN, INTERPRÈTE}

En premier lieu, il convient de faire précéder à l'analyse d'un certain nombre de textes de Morin un survol des premières années de son activité pianistique 3 , de façon à démontrer comment sa formation d'interprète a pu déterminer ses éventuelles prises de position face à la musique française, à ces compositeurs qui lui sont, pour la plupart, contemporains, et auxquels il consacre bientôt des chroniques ; le langage musical de certains d'entre eux présente certes quelques éléments de traditionalisme, mais les noms qui ont le plus retenu son attention demeurent ceux ayant pleinement participé de l'esthétique moderne.

Sa rencontre avec ce mouvement moderniste remonte principalement au temps de ses études de piano à Paris, suite à l'obtention du Prix d'Europe en 1912. Élève du maître Ricardo Viñes (1875-1943) - qui s'était vu confié, entre autres, la création de pièces pour piano de Debussy telles que L'île joyeuse -, Morin s'initie aux particularités, aux qualités de cette musique et en fait progressivement son répertoire de prédilection qui s'articule bientôt autour de la musique de Debussy et de Ravel.

Ayant été admis au salon littéraire parisien de M. et Mme Charles de Pomairols ${ }^{4}$, Morin fait, au cours de son premier voyage d'étude (1912-1914), la connaissance de compositeurs d'orientations esthétiques variées, plongeant, par la même occasion, dans l'effervescence de la vie musicale de la métropole. Par exemple, en plus de rencontrer le mélodiste Reynaldo Hahn (1874-1947), il a « [...] l'honneur de jouer plusieurs fois chez Gabriel Fauré qui l'[encourage] ${ }^{5}$ ». Il rencontre Maurice Ravel et Jean Cocteau $(1889-1963)^{6}$, puis s'intègre, au cours d'un second séjour (1919-1925), à l'entourage du Groupe des Six.

2 Élément clé de l'«Esthétique de la réception » formulé par Jauß à la fin de la décennie 1960, l'horizon d'attentes consiste en la capacité d'un individu à anticiper et à comprendre une œuvre en faisant appel à tout un système de référents acquis au contact de sa culture propre. Une lecture critique de la théorie de la réception de Jau $\beta$ et de l'histoire des systèmes similaires peut être trouvée dans l'ouvrage de Marchand, 2003.

3 Pour une étude approfondie de la carrière pianistique de Léo-Pol Morin, voir : Caron, 2009.

4 Ibid., pp. 63-64.

5 W. Chevalier. Le Soleil. 1935. "Léo-Pol Morin nous parle de ses maîtres ». 5 mars. (Cité dans Caron, 2009, p. 169.)

6 Ibid., p. 62. 
En 1914, Morin est forcé de rentrer au Canada en raison du déclenchement de la Première Guerre mondiale. Au pays, il poursuit sa pratique pianistique et commence tôt à donner des concerts qui ne passent pas inaperçus, contribuant à provoquer ce que l'on pourrait nommer la «Crise du futurisme». Par exemple, suite à un concert qu'il donne en compagnie de la basse Arthur Laurendeau le 17 avril 1917, au programme duquel figurent des œuvres de Debussy et de Ravel, un dénommé Pierre Nisard écrit dans le journal étudiant L'Escholier:

Quel souci de ces règles [de l'écriture musicale] MM. Debussy et Ravel montrent-ils dans le Paon, le Vent d'ouest et le chant des petits enfants. Leur système même s'y oppose. L'harmonie seule, et encore, forme la base de leur méthode. Voici que j'entends de magnifiques accords, un soupçon de mélodie commence à me chatouiller le tympan, mon imagination monte à petits pas vers le rêve. Pan! un plaqué formidable me fait dégringoler jusqu'au sous-sol de la clef de fa. Parfois les alliances de sons deviennent si joliment criardes, d'un faux si désespérant que les auditeurs éclatent de rire. ${ }^{7}$

Les détracteurs de la musique moderne font toutefois face à la plume d'un Léo-Pol Morin convaincu, qui répond volontairement et sans demi-mesure, bousculant le conservatisme de la critique musicale québécoise. Il écrit notamment dans Le Devoir:

Ces braves réactionnaires, incapables d'aimer l'art, ils aspirent à pleins poumons à retomber en enfance... putréfiés déjà, poussière... Croyez-moi, il n'y a [...] d'antimusical et d'antiartistique que votre manière d'ignorer et par conséquent de ne pas comprendre l'école moderne. ${ }^{8}{ }^{»}$

Aussi, les meilleurs indices du rôle que peuvent avoir joué les programmes de Morin dans l'intégration de la musique moderne en sol québécois se trouvent-il dans les critiques de ses concerts, où se rencontrent à ce sujet nombre de commentaires élogieux ${ }^{9}$. Les prestations qu'il donne au cours de la décade suivante s'inscrivent dans ce même esprit de diffusion de la musique du jeune $\mathrm{XX}^{\mathrm{e}}$ siècle, conférant une visibilité propre à la musique de compositeurs tels que Debussy ${ }^{10}$, Ravel ${ }^{11}$, Schönberg et Bartók ${ }^{12}$. Ces concerts furent autant d'occasions pour Morin de parfaire l'éducation de l'auditoire et de communiquer avec eux au sujet de ces compositeurs. Un compte-rendu de ces événements musicaux faisait ensuite l'objet de textes publiés dans les journaux.

7 P. Nisard. L'Escholier. 1917. «Chronique musicale ». 27 avril. (Cité dans Caron, 2009, p. 85.)

8 Léo-Pol Morin. Le Devoir. 1917. «Une lettre de M. Léo-Pol Morin », Le Devoir, 7 avril 1917. (Cité dans Caron, 2009. p. 80.)

9 La thèse de doctorat de Claudine Caron présente quelques-unes de ces critiques. Voir les pages 76 et 338 .

10 Il s'agit d'un concert hommage à Debussy ayant eu lieu le 11 décembre 1927.

11 Morin participe à deux tournées de Maurice Ravel. Il l'accompagne au cours de sa tournée européenne, en 1923, et participe à un concert qui a lieu à Montréal au cours de la tournée américaine du compositeur, le 19 avril 1928.

12 Morin donne un concert-causerie sur les musiques autrichienne et hongroise, en mars 1927. La causerie du concert donne lieu à un article paru dans le journal La Patrie : 1927. "Bartók contre Schoenberg ». 19 mars. 


\section{LÉo-Pol Morin, Musicographe}

Les écrits musicographiques de Léo-Pol Morin se retrouvent sous trois formes. D'une part, sous la forme élémentaire des rubriques qu'il publia dans les journaux, où alternent des textes tantôt consacrés à l'actualité du concert - il agit alors véritablement à titre de critique -, tantôt faisant œuvre d'éducation et abordant des sujets aussi divers que certains compositeurs ciblés, la musique canadienne, le rôle du critique et l'éducation du public. D'autre part, une sélection de ses textes se trouve colligée dans deux anthologies datant respectivement de 1930 et de 1944. La première contient une révision d'articles publiés dans le journal La Patrie, alors que la seconde consiste en une collection des écrits du critique faite par ses amis à titre posthume, où se retrouvent parfois plusieurs versions d'un même texte, permettant l'observation de l'évolution de la pensée esthétique du musicien. L’objectif avoué de ces lignes étant de souligner l'aspect pédagogique de ces textes, ne seront ici retenus que les articles de journaux, de sorte que le matériel à l'étude sera celui avec lequel le public fut en contact. Les seuls extraits tirés des anthologies concernent le rôle du critique, sujet de second niveau ne nécessitant pas une prise de position ancrée dans l'actualité des événements de l'époque.

L'aspect musicographique des publications du musicien a fait l'objet du mémoire de maîtrise de Claire Villeneuve, déposé à l'Université de Montréal en $1975^{13}$. Consacrant principalement son étude aux articles de Morin traitant de musique canadienne, elle opère une synthèse révélatrice des textes où il est question de la place accordée à l'art musical dans la société québécoise. Villeneuve présente un inventaire complet des articles de Morin et met en relief certains articles où le musicographe réclame que soient reconsidérées les méthodes d'enseignement de la musique, de sorte que le bagage de connaissances possédé par l'auditeur au moment de vivre l'expérience musicale le dispose à en apprécier les plus élémentaires qualités. Pour Morin, l'inculture musicale est le fruit d'un système d'éducation défaillant qu'il faut réévaluer. Il prône la création d'un Conservatoire gratuit, idéalement calqué sur le modèle européen, dont les programmes destinés aux interprètes dispenseraient une formation théorique complète.

Conscient que ses requêtes ne pouvaient trouver écho que dans un avenir relativement éloigné ${ }^{14}$, Morin s'est investi personnellement d'une mission pédagogique ayant caractérisé l'ensemble de ses activités musicales. Dès l'automne 1927 , il propose des cours de groupe d'initiation à la musique ${ }^{15}$, de façon à réduire les coûts d'inscription individuels et, par le fait même, à rendre son enseignement accessible au plus grand nombre.

Dans ses rubriques journalistiques, non seulement revendique-t-il le développement du milieu musical montréalais, mais il consacre aussi de nombreux articles à la prospection de la vie et de l'œuvre de compositeurs ciblés ou, plus largement, à la musique moderne. Il importe ici d'inscrire Morin au cœur

13 Villeneuve 1975.

14 De fait, le Conservatoire de Montréal n'est fondé qu'en 1942.

15 Léo-Pol Morin. La Patrie. 1927. «Les cours du pianiste Léo-Pol Morin ». 24 novembre. 
d'une tradition de musiciens actifs non seulement à titre de critiques, mais aussi de musicographes et de compositeurs, qui œuvrèrent à la diffusion du savoir et à l'émergence d'une vie musicale riche en sachant tirer profit de la visibilité conférée par leurs positions privilégiées. Dans sa thèse de doctorat, Claudine Caron a mis en évidence une succession de littérateurs de la musique s'étant engagé dans la critique et la revendication du développement de la vie musicale de la province ${ }^{16}$. Les noms les plus connus et les mieux documentés à ce jour, outre Morin, sont ceux de Guillaume Couture ${ }^{17}$ (1851-1915), de Jean Vallerand $(1915-1994)^{18}$ et de Serge Garant ${ }^{19}(1929-1986)$. Il conviendrait d'étendre cette idée au discours musicographique général, en considérant aussi la portée pédagogique que peuvent avoir eu les écrits de tous ces acteurs importants de notre vie musicale, lesquels renseignent les lecteurs sur la vie de compositeurs clé, traitent de l'importance historique d'œuvres ciblées ou revendiquent le droit de cité des langages avant-gardistes les plus déroutants. Ainsi ces hommes de fortes convictions contribuèrent-ils à éveiller et à enrichir une conscience proprement musicale, faisant émerger chez leurs lecteurs une liberté de penser fondée sur une réelle compréhension du fait musical.

Dans le cas de Morin, les compositeurs français qui lui sont contemporains constituent le cœur de ses chroniques et ce, dès le début de sa carrière. Le nombre d'articles qui leur sont consacrés ne manque pas d'attirer l'attention. Déjà, dans Le Nigog, il publie des articles traitant de Fauré (octobre), de Ravel (novembre) et de Dukas (décembre). Aussi, ceux parmi les textes rédigés au cours de sa carrière, portant sur les compositeurs de ce pays, qui furent colligés dans l'anthologie Musique occupent-ils le quart du volume dans son ensemble, soit 111 pages sur un total de 440 .

En 1918, son implication dans la fondation de la revue artistique d'avant-garde Le Nigog, lui vaut une tribune régulière où faire valoir ses opinions et intérêts. Il y affiche clairement ses positions, et affirme qu'il souhaite secouer «les préjugés, [...] contrer l'inculture et [...] faire reconnaître auprès de la société québécoise la nécessité de s'ouvrir à la création artistique ${ }^{20} »$.

\section{Disposition de l'auditoire face à la musique de la modernité}

Avant d'entreprendre l'analyse des écrits journalistiques de Morin, il convient de s'intéresser à l'idée qu'il se fait de l'état de l'auditoire de son temps et de questionner ce qu'il considère être la meilleure façon de pratiquer la critique musicale en son pays. Outre deux sections portant sur les écoles nationales et sur la musique canadienne, l'anthologie Papiers de musique contient un

\footnotetext{
16 Caron 2099, pp. 394-396.

17 Lefebvre. 2004.

18 Lefebvre 1996.

19 Lefebvre 1986.
}

20 La rédaction. Le Nigog. 1918. "Signification », janvier, pp. 2-4. Dans les rubriques qu’il tient au cours de la seule année d'activité du Nigog, Morin fait déjà appel aux principaux arguments employés tout au long de sa carrière journalistique. Les livraisons de cette revue s'étant interrompues à la fin de l'année 1918, c'est au journal La Patrie que Morin fait à nouveau valoir, à partir de 1926, la prime importance du caractère humain et de la vie dans la musique. 
important chapitre dont le titre en lui-même est fort révélateur : "Coups de poing dans la neige ». On retrouve dans tous ces écrits les opinions de Morin quant au métier et au rôle du critique musical ainsi que sa vision des dispositions du public québécois de l'époque face à la musique. Un article publié dans le journal Le Canada (1935) témoigne de l'état précaire du goût musical où se complaisent les auditeurs avares de virtuosité et peu soucieux des véritables vertus de l'art:

Il y a déviation du sens musical. On aime la virtuosité ou la romance, on aime le tour de force réussi sans peine, on aime l'acrobatie et la flatterie des sens mais, au fond, très peu le mystère musical, la révélation de l'âme. À cela, la plupart des auditeurs restent fermés, car ce mystère demande une certaine initiation. [...] Initiation et non pas entraînement spécialisé. ${ }^{21}$

Cette initiation, qui est précisément la base minimale des connaissances qu'il réclame lorsqu'il revendique la rénovation du milieu de l'enseignement de la musique, Morin l'offre à ses lecteurs par le biais de ses écrits. Aussi attribuet-il au critique la responsabilité d'éveiller les mélomanes aux valeurs profondes de la musique; cette hypothèse est d'ailleurs confirmée par le tout premier article qu'il publie au journal La Patrie, en 1926, où il écrit qu'il souhaite «éveiller la curiosité des amateurs de musique, développer le goût des belles œuvres et aider, le cas échéant, certains talents encore timides à se préciser ${ }^{22} »$. Dans l'anthologie Papiers de musique se trouvent aussi un certain nombre de commentaires corroborant cette idée. D’une part, Morin y écrit qu'il «[...] considère le rôle du musicographe comme double: il est à la fois l'informateur et l'éducateur du public ${ }^{23}$ ». Quant à la critique musicale, c'est en ces termes qu'il en recommande la pratique:

La critique subjective est [...] la seule qui ait quelque chance de se développer sur notre territoire jusque dans un avenir éloigné. Elle n'est d'ailleurs pas mauvaise et nous savons qu'elle peut découvrir autant que l'autre [scientifique, objective], les secrets d'une œuvre, son ordonnance, sa forme et son essence même. ${ }^{24}$

Cette subjection faisant appel aux sens et à la vie dans la musique ${ }^{25}$, Morin la retrouve précisément dans la musique des compositeurs français au service de laquelle il met principalement ses talents d'interprète. C'est pour cette raison qu'il fera constamment référence à ce répertoire dans les diverses facettes de ses activités visant l'initiation du grand public aux plaisirs de la modernité musicale.

À travers ses écrits, on prend conscience de l'ouverture lente mais progressive des auditeurs confrontés à la nouvelle musique. L'humeur de Morin alterne

21 L.-P. Morin. Le Canada. 1935. « Pourquoi aimons-nous la musique ». 2 février.

22 L.-P. Morin. La Patrie. 1926. «Préliminaire ». 6 mars.

23 L.-P. Morin, Papiers de musique, p. 10.

24 Ibid., p. 168.

25 Voir aussi l'article suivant : L.-P. Morin, «La musique, plaisir des sens » (Le Canada. 1935. 9 février). 
certes entre des pôles extrêmes d'optimisme et de découragement, mais il semble in fine garder confiance en l'auditeur ${ }^{26}$. Ainsi, alors qu'à l'époque du Nigog il s'en prend à la superficialité des auditeurs qui préfèrent d'insipides épanchements de virtuosité à la reconnaissance du mérite d'un interprète dévoué à la réelle mise en valeur de la musique ${ }^{27}$, il rédige, en 1922, une note passablement optimiste à l'intention du journal français Le Matin:

Mes programmes ont été partout bien accueillis, la presse a été généreuse, chaleureuse, et je suis revenu en France avec la conviction très ferme que l'on aime décidément le piano dans la province de Québec! Mais tout est relatif, et un pianiste étranger, à ma place, eut quitté le Canada bien avant moi et dans un état voisin de la prostration. ${ }^{28}$

Mais que les auditeurs aiment la musique ne signifie pas nécessairement qu'ils la comprennent tout à fait; on trouve d'ailleurs des indices de cette réalité dans deux articles écrits par Morin ${ }^{29}$ entourant un concert donné par Maurice Ravel à Montréal le 19 avril 1928, où, bien qu'il déplore l'accueil ambigu réservé au célèbre compositeur par le public — qui, peut-être, s'attendait à de nouvelles prouesses de virtuose ${ }^{30}$ - il maintient que l'auditoire se montra malgré tout enthousiaste. Dans sa monographie sur Rodolphe Mathieu, MarieThérèse Lefebvre rapporte toutefois qu'étant «las de se battre contre une inertie lourdement cimentée dans le paysage culturel québécois ${ }^{31} »$, Morin quitte le Québec le 21 mai 1929.

C'est notamment en raison d'une convalescence qui se prolonge jusqu'en octobre 1930 que Léo-Pol Morin demeure en Europe au cours de l'année suivante. C'est à ce moment qu'il révise les articles publiés précédemment et qui seront regroupés dans l'anthologie Papiers de musique, où figure un texte où il réaffirme avec force conviction que les goûts des auditeurs peuvent s'élargir. Il croit

[...] la masse susceptible de prendre plaisir à la musique sérieuse et savante, et même "moderne", à la condition que celle-ci ne néglige pas les sens et ce don qu'il est convenu d'appeler le charme, qu'elle ait de l'intérêt dramatique, en un mot, de la vie. Dans ce cas, le grand public peut goûter une page de Debussy ou de Strawinsky tout aussi bien qu'une autre de Bach, de Grétry ou de Massenet. ${ }^{32}$

26 Morin écrivit de nombreux article destinés à défendre un public qui, bien que de bonne volonté, ne peut malheureusement se soustraire aux carences de son éducation musicale. Le lecteur pourra, à titre d'exemple, consulter les articles suivants : «Le public et la musique » (La Patrie, 15 mai 1926) et "Le goût de l'art» (La Patrie, 20 novembre 1926).

27 L.-P. Morin. Le Nigog. 1918. " Le public des concerts ». Avril, p. 127.

28 L.-P. Morin. Le Matin. 1922. "Rétrospection », 10 juin. (Cité dans Caron 2009, p. 140).

29 Voir les articles « Maurice Ravel " (La Patrie. 1928. 7 avril) et « En marge du concert de Maurice Ravel », (La Patrie, 1928. 21 avril).

30 « Maurice Ravel, ce grand artiste qui ne casse rien, qui n'est pas un virtuose [...] ne vaut rien pour les amateurs de pianos écrasés. Il lui manque le coup de main, le coup de tête, les gros muscles du cou, de longs cheveux tirés, une mine langoureuse à l'occasion, pensive et désespérée. Il lui manque quelques-uns de ces ressors, de la force, de ne pouvoir faire à lui tout seul grand tapage. » (L.-P. Morin. La Patrie. 1928. « En marge du concert de Maurice Ravel ». 21 avril.)

31 Lefebvre 2055, p. 178.

32 L.-P. Morin. La Presse. 1930. "Défense du public », 1 février. 


\section{Une trinité française}

Parmi tous les compositeurs qui firent l'objet de l'une des chroniques hebdomadaires de Morin, il s'en trouve trois qui pourraient être rassemblés sous la forme symbolique d'une trinité : Gabriel Fauré, Claude Debussy et Maurice Ravel. Le critique voit en ces musiciens les rénovateurs de l'art musical français 33 , les premiers à composer une musique qui convienne au « génie de la race $^{34}$ ", une musique qui sache se soustraire aux excès du wagnérisme, aux débordements romantiques et à l'italianisme, mouvements dont l'activité créatrice avait supplanté l'esthétique proprement française des grandes scènes parisiennes du XIX ${ }^{\mathrm{e}}$ siècle. Il traite notamment de la question dans un texte qui se retrouve dans l'anthologie Musique 35 .

Dans son discours sur les trois compositeurs, il fait appel à une ingénieuse démarche rhétorique - certains articles de Jean Vallerand feront état d'une démarche similaire ${ }^{36}$. En faisant appel à un champ lexical coloré et sensuel, Morin influence la réceptivité des auditeurs ${ }^{37}$ en les disposant favorablement à cette musique par le biais de préjugés positifs. Ainsi peut-on lire la description suivante de la musique de Fauré dans un article publié en 1930:

C’est un art de poésie, d'images et de formes sensibles. C'est un art de sentiment, de nuances subtiles, et qui ne choque jamais. Un art tendre, délicat, mais vigoureux aussi. Des harmonies chaudes, mais discrètes, séduisantes et fuyantes, ombrent avec un extrême raffinement la tonalité générale de cette musique..$^{38}$

\section{Ses observations de la musique de Debussy sont similaires :}

Il a cherché et trouvé cette concordance mystérieuse qu'il y a entre la nature et le cerveau de l'artiste. Il a libéré la musique par la nature. Au lieu d'être une froide spéculation arithmétique, scientifique ou topographique, la musique, avec lui, est devenue plus sensible. En obéissant aux lois

33 À ce sujet, voir la série de cinq textes consacrés à la musique de Debussy et de ses compatriotes français dans l'anthologie Musique, pp. 109-168.

34 L.-P. Morin, Musique, p. 186.

35 Ibid., p. 116.

36 «La connaissance commence par l'amour et comment aimer autre chose que la vie? Le secret de l'intimité avec le beau contemporain est peut-être dans cette formule. En d'autres termes : se méfier des œuvres qui ne vivent pas. " (Jean Vallerand. Le Devoir. 1953. "Comment accéder au beau contemporain ». 21 mars.) À titre d'exemple, le lecteur pourra aussi consulter la monographie Introduction à la musique (1949) de Vallerand, ouvrage d'initiation destiné au jeune public, ainsi que de nombreux articles où celui-ci adopte une perspective semblable de manière à élever la musique au niveau d'un art capable d'influence sur l'ascèse morale de l'homme moderne. Les articles suivants pourront être consultés à titre d'exemples : "Le problème crucial " (Montréal-Matin. 1947. 30 octobre), "Positions de la musique contemporaine " (Le Devoir. 1952. 16 août) et «Idées anciennes sur la musique moderne " (Le Devoir. 1955. 29 janvier). La monographie de Marie-Thérèse Lefèvre consacrée à Jean Vallerand contient aussi une liste essentielle de ses écrits, répertoriés par catégories.

37 La réceptivité peut ici être associée au processus d'interprétation esthésique, lequel est l'une des trois dimensions du phénomène symbolique. La dimension esthésique procède de l'attribution d'une signification à la forme par le récepteur ou, plus simplement, de la construction d'une signification « [...] en un processus actif de perception». (Voir Nattiez 1987, p. 32.)

38 L.-P. Morin. La Presse. 1930. « Gabriel Fauré ». 15 novembre. 
secrètes de la nature et des vibrations naturelles, elle est mieux proportionnée à notre échelle humaine. ${ }^{39}$

Enfin, c'est toujours par ce vocabulaire sensuel que sont caractérisés les commentaires concernant la musique de Ravel: «Il m’a toujours paru difficile de résister au sortilège de cette musique si fine, subtile, incisive, pénétrante, lumineuse, chaude aussi, de Ravel $40_{\text {» }}$

Les trois exemples précédents démontrent bien la disposition favorable à laquelle Morin convie le lecteur. La même démarche discursive peut toutefois servir des causes bien différentes ; en ce sens, il importe de souligner la différence flagrante qu'il existe entre le lexique que Morin réserve à la présentation des compositeurs français et celui auquel il fait appel lorsqu'il s'intéresse à la musique des compositeurs germaniques modernes, lesquels sont fort peu représentés dans ses propres programmes. Ainsi peut-on lire sous sa plume que «les théories de Schönberg sont aussi nombreuses et troublantes que ses œuvres» et que "quelques phrases mélodiques aux contours accidentés, quelques harmonies étudiées au microscope suffisent à [y] exprimer tout un drame ${ }^{41}$ ». Il est aussi question de "[...] la musique cérébralement compliquée d'Alban Berg [et de] celle, impérieuse ${ }^{42}$, d'Hindemith [...] ${ }^{43}$ ». Il semble ici évident que si la chaleur du ton invite à la découverte du premier corpus, les termes dont le critique fait usage afin de décrire la musique des compositeurs germaniques invitent surtout à une distance respectueuse.

\section{Perspectives pour une étude des périodiques}

Jusqu'à présent, ces lignes ont été consacrées à l'étude de la démarche du critique, dont le but était d'offrir au public une perspective éclairée au sujet de la musique de la modernité. Or, il est un second degré de réception sur lequel Morin ne peut avoir eu de contrôle : celui de la réception de ses propres textes. L'hypothétique portée du contenu de ses écrits auprès des mélomanes dans un contexte de diffusion donné devra encore faire l'objet de nouvelles investigations avant que des conclusions définitives puissent être formulées quant au rôle joué par Léo-Pol Morin dans l'intégration et la diffusion de nouveaux répertoires sur la scène musicale québécoise au début du siècle dernier.

Un certain nombre d'informations portant sur les journaux dans lesquels les textes de Morin étaient publiés, à l'intérieur de plus vastes sections consacrées à la vie culturelle, peuvent aider le lecteur à mieux comprendre le contexte de diffusion de sa production journalistique. Les quelques données présentées ici pourront ultérieurement servir de point de départ à une investigation plus approfondie des périodiques ciblés. cembre.

39 L.-P. Morin. La Patrie. 1927. «Claude Debussy-Essai d'esthétique d'après ses notes ». 3 dé-

40 L.-P. Morin. Le Canada. 1938. « Ravel est mort, vive Ravel! ». 3 janvier.

41 L.-P. Morin. La Patrie. 1927. "Bartók contre Schoenberg ", 19 mars.

42 Ce texte est repris dans l'anthologie Papiers de musique, où le terme «impérieuse " est remplacé par « autoritaire ", terme qui suggère une certaine austérité.

43 L.-P. Morin. La Presse. 1930. "Musiques nationales ». 31 mai. 
Contrairement au lectorat possiblement restreint dont peut avoir bénéficié une revue avant-gardiste telle que Le Nigog, les journaux qui accueillent par la suite les fruits de la plume du musicien sont tous des journaux libéraux 44 à grand tirage. Il faut savoir qu'au départ, les articles étaient très courts. Au temps de La Patrie, les rubriques accordées à Morin n'étaient que d'une colonne. Situé entre les pages vingt-huit et quarante de la livraison du samedi qui en comptait soixante, le texte se retrouvait à l'extérieur de la page gauche ${ }^{45}$. Le tirage de ce journal devait approcher trente mille copies ${ }^{46}$ à l'époque à laquelle Morin y collabora.

Le volume des articles augmente à deux colonnes lorsque Morin passe à $L a$ Presse en $1929^{47}$. La rubrique musicale était située dans les dix dernières pages des livraisons du samedi qui en comptait environ quatre-vingt. Ces rubriques faisaient suite à la lecture de la Bourse, des publicités, des sports, des annonces classées et de la nécrologie! Il s’agit, de loin, du journal ayant profité du plus important tirage ${ }^{48}$.

Enfin, lorsque Léo-Pol Morin se voit confié, en 1933, la rubrique du journal Le Canada, ce sont des articles à fréquence variable qui lui sont accordés. Le format est encore augmenté par rapport à celui de La Presse, (ici, environ trois colonnes) 49 et la rubrique se retrouve dans les toutes premières pages du journal. Dans ce journal ayant «[...] toujours été à l'avant-garde de l'opinion libre ${ }^{50} »$, le faible tirage ${ }^{51}$ ainsi que le fait que les publications aient alterné irrégulièrement entre le lundi, le mardi et le samedi pourraient toutefois avoir eu un impact négatif sur la réception des articles.

Il serait difficile, voire risqué, de spéculer davantage sur le sujet. Il est toutefois possible de supposer que ce n'est pas tant dans les écrits de Morin luimême que l'on pourra trouver des pistes de réponses quant à l'impact réel de son travail, que dans ceux de ses successeurs. Le répertoire ayant fait l'objet des écrits de chroniqueurs de la même génération que Morin ou qui lui ont succédé pourrait, entre autre, fournir des indices quant au degré d'ouverture progressif de la critique face à la musique du nouveau siècle. À titre d'exemple, il pourrait être intéressant de comparer les écrits de Morin à ceux de Jean Vallerand ${ }^{52}$ qui, à sa façon, s'est montré préoccupé par des sujets de la modernité similaires à ceux qui ont intéressé son prédécesseur ${ }^{53}$. Un compositeur tel que

44 Toutes les informations présentées ici au sujet des journaux pour lesquels travaille Morin sont tirées de Beaulieu et Hamelin 1965.

45 Voir l'annexe 1.

46 La Patrie tirait 19734 copies en 1914 et 31811 copies en 1933. (Beaulieu et Hamelin 1965, p. 135.)

47 Voir annexe 2.

48 La Presse tirait 121085 copies en 1913 et 147074 copies en 1940. (Beaulieu et Hamelin 1965, p. 142.)

49 Voir annexe 3.

50 Ibid., p. 66.

51 Le Canada tirait 18 ooo copies en 1905 et 15242 copies en 1940 (Beaulieu et Hamelin 1965, pp. 65-66).

52 Celui-ci prend la relève de Morin au journal Le Canada suite à la mort accidentelle de ce dernier en 1941.

53 Tout au long de sa carrière de journaliste, Jean Vallerand s'est montré consacré à la diffusion des concepts des courants musicaux du XX ${ }^{e}$ siècle. Passionné par la modernité musicale-notamment 
Stravinsky se voit de mieux en mieux compris et accepté par la critique ${ }^{54}$, mais le temps passe et certains facteurs de communication (la vitesse de diffusion de l'information, l'accès à l'information en temps de guerre, l'impact de la radio, la facilité grandissante du voyage, etc.), sans négliger le rôle de l'intérêt pour les sujets abordés, peuvent contribuer à créer un retard par rapport à l'actualité la plus immédiate. À titre d'exemple, il peut être observé que si Morin, déjà, en octobre $1937^{55}$, consacre tout un article à cette Jeune France de laquelle se réclame Olivier Messiaen (1908-1992), Vallerand, dans sa monographie intitulée Introduction à la musique, datée de 1949, ne fait aucunement référence au désormais célèbre compositeur français qui, déjà à cette époque, avait bouclé son «triptyque de Tristan», constitué du cycle Harawi (1945), de la Turangâlîla-Symphonie (1946-1948) et des Cinq Rechants (1948). Il sera toutefois laissé aux soins des prochaines recherches de définir avec plus de précision, comme dans le cas de la réception des chroniques de Morin en général, les facteurs qui influencèrent la rétention privilégiée de certains répertoires dans la pratique courante de la musique.

\section{Conclusion}

En substance, il peut être dit que les pratiques musicales de Léo-Pol Morin, à la fois à titre de critique musicographe, d'interprète et de pédagogue, ont sans aucun doute contribué à initier une partie de l'auditoire québécois aux corpus des compositeurs français de la modernité. Riche de son expérience en sol européen et de ses nombreux contacts de premier ordre parmi les personnalités importantes du milieu musical français, Léo-Pol Morin a su parler, avec une érudition et une éloquence certaine, de l'actualité musicale de son époque. Sa connaissance et sa compréhension des mélomanes québécois lui ont permis de mettre à profit, dans un contexte de conservatisme culturel, un réel intérêt pour la musique française en peignant la sensualité des courbes modernes par la suave subjectivité d'un verbe mis au service de la rhétorique.

Avec les trois maîtres [...] Fauré, Debussy, Ravel, la musique française prend une forme complète. Elle trouve ici son cœur, son corps, son esprit. ${ }^{56}$

celle de Stravinsky et de Honegger-il s'est graduellement ouvert à la musique contemporaine. Il s'est fortement intéressé à la musique canadienne et a questionné le rôle du compositeur-et de la musique en général-à titre de phare de la moralité au cœur d'une époque vivement marquée par les horreurs de la Guerre.

54 Dans les écrits de Vallerand, ce compositeur devient même l'incontournable figure de référence du compositeur moderne. Dans son Introduction à la musique (1949), il le qualifie même de «prophète ». (Vallerand 1949, p. 269.) Le lecteur pourra consulter l'ouvrage de Marie-Thérèse Lefebvre où sont bien tracées les grandes lignes du discours de Vallerand.

55 L.-P. Morin. Le Canada. 1937. " "Jeune France" ». 11 octobre.

56 L.-P. Morin, Musique, p. 168. 


\section{BibLIOGR APHIE}

\section{Articles de presse}

\section{Le Nigog}

La rédaction. 1918. «Signification ». Le Nigog, $\mathrm{n}^{\circ} 1$ (janvier).

Morin, Léo-Pol. 1918. « Le public des concerts ». Le Nigog, $\mathrm{n}^{\circ} 4$ (avril).

\section{La Patrie}

La Patrie. 1926. « Préliminaire ». 6 mars.

La Patrie. 1926. «Le public et la musique ». 15 mai 1926.

La Patrie. 1926. "Le goût de l'art». 20 novembre.

La Patrie. 1927. «Le Groupe des Six ». 22 janvier.

La Patrie. 1927. "Bartók contre Schoenberg ». 19 mars.

La Patrie. 1927. «Les cours du pianiste Léo-Pol Morin » 24 novembre.

La Patrie. 1927. "Claude Debussy - Essai d'esthétique d'après ses notes ». 3 décembre.

La Patrie. 1927. «Des fleurs qui tuent ». 22 octobre.

La Patrie. 1928. « Maurice Ravel». 7 avril.

La Patrie. 1928. «En marge du concert de Maurice Ravel». 21 avril.

\section{La Presse}

La Presse. 1930. «Défense du public ». 1 février.

La Presse. 1930. "Musiques nationales ». 31 mai.

La Presse. 1930. «Paul Dukas ». 16 août.

La Presse. 1930. «Gabriel Fauré ». 15 novembre.

\section{Le Canada}

Le Canada. 1933. "Claude Debussy et la musique française d'aujourd'hui». 18 décembre.

Le Canada. 1935. «Pourquoi aimons-nous la musique ». 2 février.

Le Canada. 1935. "La musique, plaisir des sens ». 9 février.

Le Canada. 1937. " "Jeune France" ». 11 octobre.

Le Canada. 1938. "Ravel est mort, vive Ravel! ». 3 janvier.

Le Canada. 1938. «La grande pitié de notre éducation musicale ». 19 septembre.

\section{Travaux universitaires :}

Caron, Claudine. 2009. "Chroniques des concerts du pianiste Léo-Pol Morin (1892-1941) : pour un portrait de la modernité musicale au Québec », Thèse de doctorat, Université de Montréal.

Marcotte, Claire. 1942. « Bio-bibliographie de Léo-Pol Morin 1892-1941 », Travail dirigé [non-édité], Université de Montréal.

Villeneuve, Claire. 1975. « Léo-Pol Morin (1892-1941), musicographe», Mémoire de maîtrise, Université de Montréal. 


\section{Monographies}

Beaulieu, André et Jean Hamelin. 1965. Les journaux du Québec de 1764 à 1964. Québec : Les cahiers de l'institut d'histoire, Presses de l'Université Laval.

Lefebvre, Marie-Thérèse. 1996. Jean Vallerand et la vie musicale du Québec-1915-1994. Méridien.

—. 2005. Rodolphe Mathieu, 1890-1962: l'émergence du statut professionnel de compositeur au Québec. Sillery : Septentrion, coll. "Cahiers des Amériques - Musique ».

-1986. Serge Garant et la révolution musicale au Québec. Montréal : Louise Courteau, éditrice.

Marchand, Guy. 2003. Bach ou la Passion selon Jean-Sébastien - De Luther au nombre d'or. Paris : L'Harmattan, coll. «Univers Musical».

Morin, Léo-Pol. 1930. Papiers de musique. Montréal.

Morin, Léo-Pol. 1944. Musique. Montréal : éd. Beauchemin.

Nattiez, Jean-Jacques. 1987. Musicologie générale et sémiologie. Mayenne : Christian Bourgois éditeur, coll. «Musique / Passé / Présent ».

Vallerand, Jean. 1949. Introduction à la musique. Montréal : Chantecler, coll. « Humanitas ».

\section{Références aux articles de Jean Vallerand}

Le Devoir. 1952. " Positions de la musique contemporaine ». 16 août.

Le Devoir. 1953. «Comment accéder au beau contemporain». 21 mars.

Le Devoir. 1955. «Idées anciennes sur la musique moderne ». 29 janvier.

Montréal-Matin. 1947. « Le problème crucial ».30 octobre.

Web

Desautels, Andrée. «Léo-Pol Morin », Encyclopédie de la musique au Canada. http://www.thecanadianencyclopedia.com/index.cfm?PgNm=TCE\&Para $\mathrm{ms}=\mathrm{Q} 1 \mathrm{ARTQ} 0002453$

\section{Varia}

Lefebvre, Marie-Thérèse. 2004. «Qu'a-t-il manqué à Guillaume Couture? Portrait d'un personnage controversé dans le milieu musical montréalais de la fin du XIX ${ }^{\mathrm{e}}$ siècle », Les cahiers des Dix, ${ }^{\circ} 58$.

Olivier, Dominique. 1996. « Jean Vallerand, critique musical», Les cahiers de l'ARMuQ, n ${ }^{\circ} 18: 11-24$.

\section{Bibliographie SÉLECTIVE DES ARTICLES DE PRESSE}

\section{Articles de presse}

\section{Au sujet de la critique musicale}

La Patrie. 1926. «Le Prix d'Europe. I. La critique et le Prix d'Europe». 5 juin.

La Patrie. 1927. «Les artistes canadiens devant la critique ». 15 janvier. 
La Presse. 1930. « Notre critique musicale». 27 décembre.

Le Canada. 1936. "La critique radiophonique ». 31 octobre.

Le Canada. 1938. "Cet animal, la critique... ». 14 février.

Le Canada. 1938. "Le jeu de la critique». 12 décembre.

Le Canada. 1940. «La critique, refuge des âmes mûres ». 8 juillet.

\section{Au sujet du public}

La Patrie. 1926. «Le public des concerts ». 22 mai.

La Patrie. 1926. «Le goût de l'art». 20 novembre.

La Patrie. 1927. « Paradoxe autour de la musique, du public et des critiques ». 23 avril.

La Patrie. 1928. «Musiciens, dilettantes et non-musiciens au concert». 10 mars. La Presse. 1930. "Défense du public ». 2 février.

Le Canada. 1934. "L'éducation par disque ». 14 juillet.

Le Canada. 1935. "Le public devant les programmes des concerts symphoniques». 30 septembre.

Le Canada. 1939. "La grande pitié de notre culture musicale ». 8 mai.

Morin, Léo-Pol. 1918. « La légende de l'art musical canadien et les musiciens de Montréal ». Le Nigog, $\mathrm{n}^{\circ} 1$ (janvier) : 17. 


\section{AnNeXe I}

\section{La Patrie : 1926-1929}

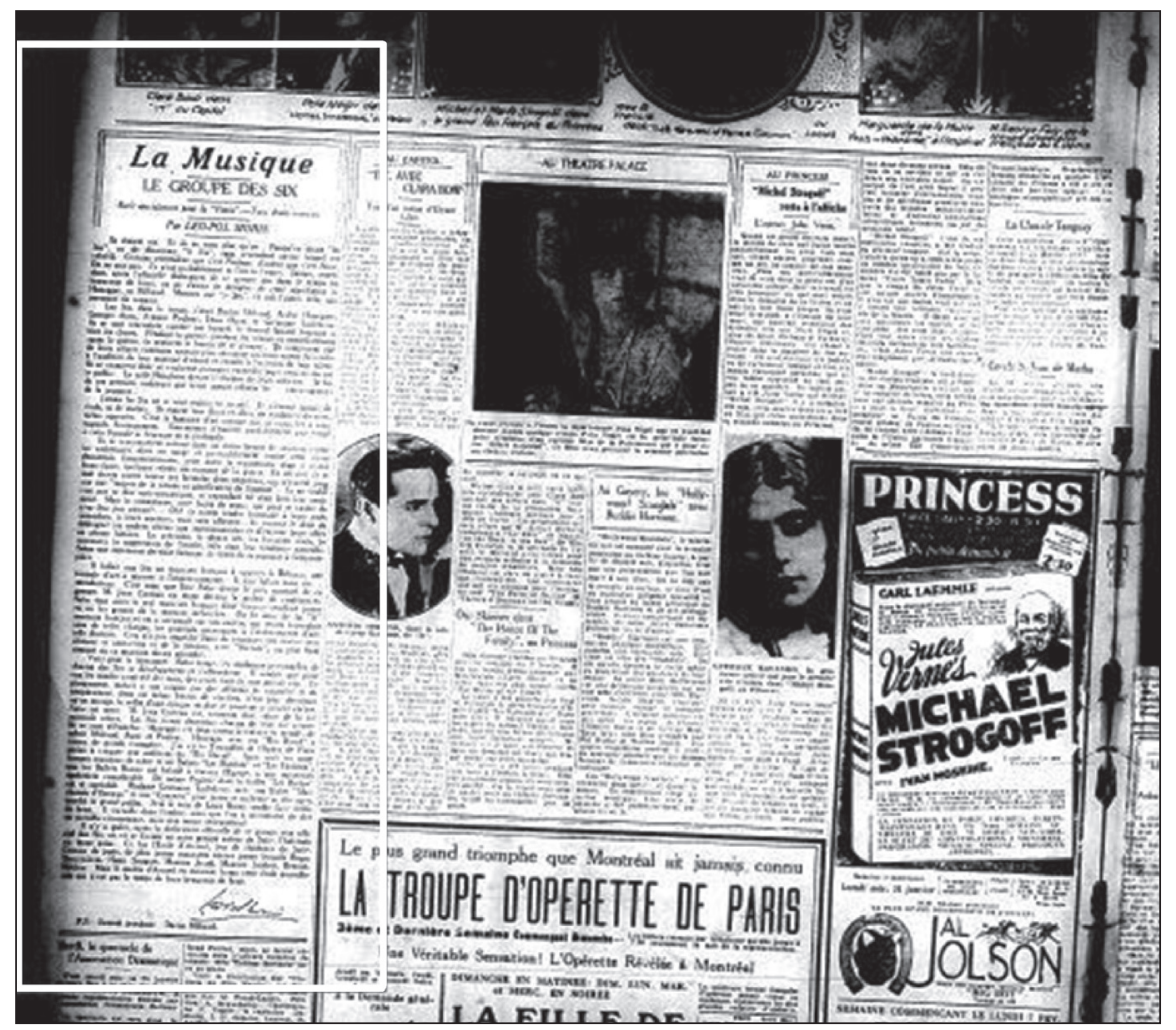

L.-P. Morin, « Le Groupe des Six », La Patrie, 22 janvier 1927.

\section{Sélection d'articles consacrés spécifiquement à des compositeurs}

La Patrie. 1927. « M. Darius Milhaud». 29 janvier.

La Patrie. 1927. "La musique de Claude Debussy ». 10 décembre.

La Patrie. 1928. « Le Roi David - Chef d'œuvre populaire d'Arthur Honegger ». 17 mars.

La Patrie. 1928. "La religions beethovenienne ». 7 janvier. 


\section{Annexe II}

\section{La Presse : 1929-1931}

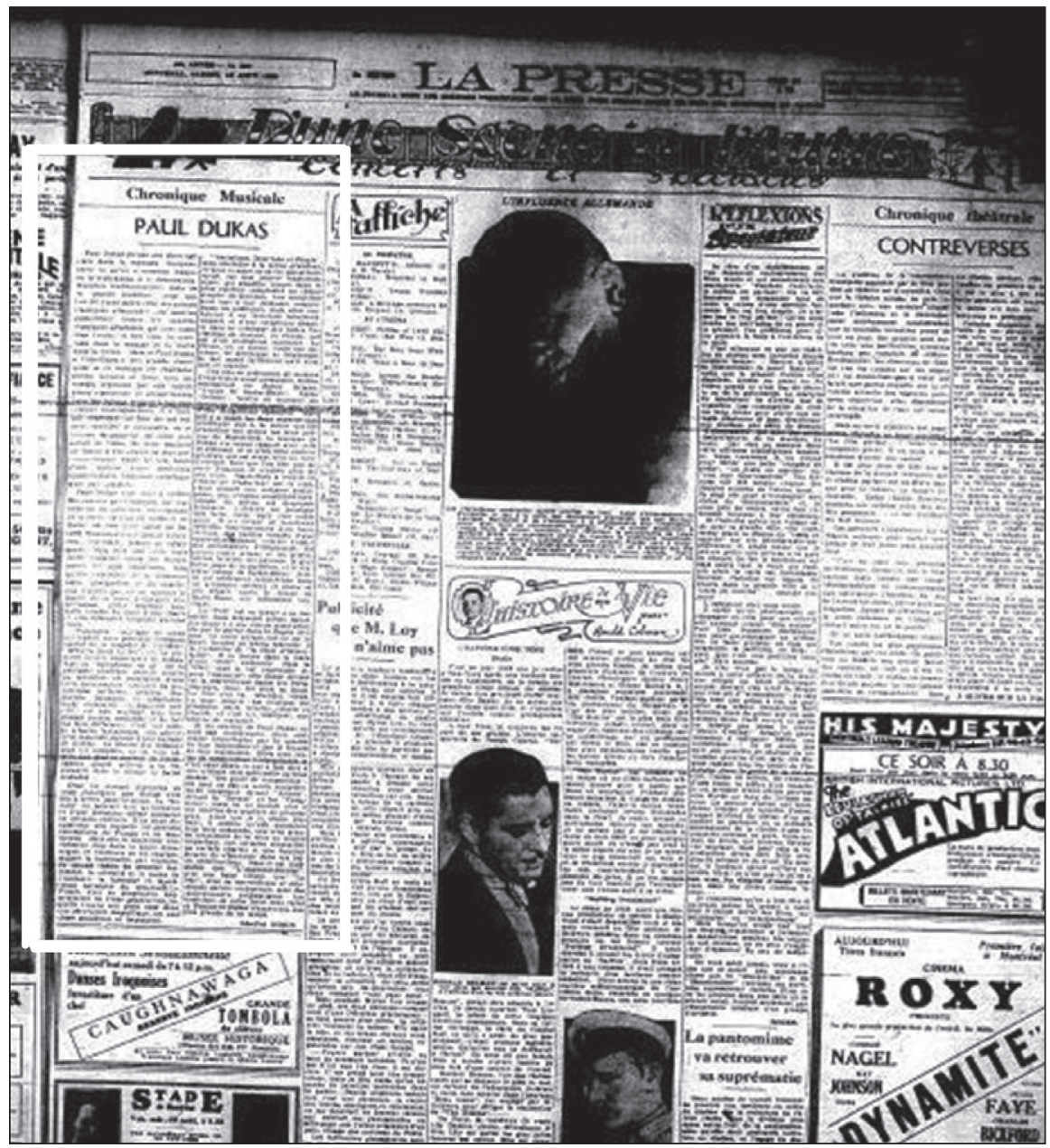

Léo-Pol Morin, «Paul Dukas », La Presse, 16 août 1930.

\section{Sélection d'articles consacrés spécifiquement à des compositeurs}

La Presse. 1930. «Les Six ont dix ans ». 25 janvier.

La Presse. 1930. «Paul Dukas ». 16 août.

La Presse. 1930. "Le brésilien Villa-Lobos ». 13 septembre.

La Presse. 1930. "Gabriel Fauré ». 15 novembre.

La Presse. 1931. «Albert Roussel ». 7 février.

La Presse. 1931. «Gounod, musicien profane ». 25 avril. 


\section{ANNeXe III}

\section{Le Canada : 1933-1941}

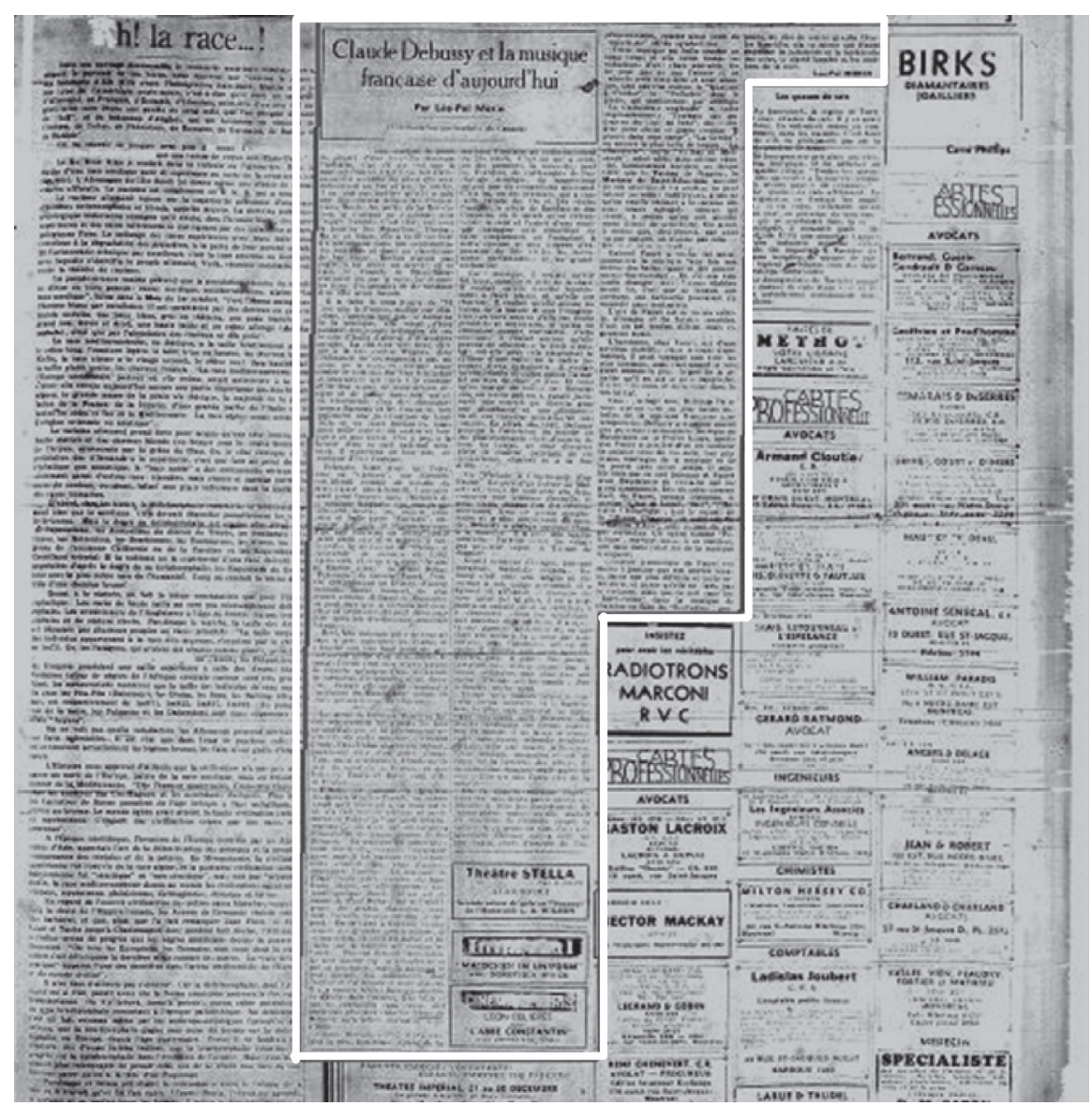

Léo-Pol Morin, «Claude Debussy et la musique française d'aujourd'hui », Le Canada, 18 décembre 1933

\section{Sélection d'articles consacrés spécifiquement à des compositeurs}

Le Canada. 1933. "Claude Debussy et la musique française d'aujourd'hui ». 18 décembre.

Le Canada. 1934. «Manuel de Falla ». 28 avril.

Le Canada. 1934. "Igor Stravinsky ». 23 juin.

Le Canada. 1937. «La musique de Vincent d'Indy ». 9 août.

Le Canada. 1937. " "Jeune France" ». 11 octobre.

Le Canada. 1938. « Les "mots" d’Erik Satie ». 25 août. 


\title{
RÉSUMÉ
}

Figure d'importance du milieu musical québécois au début du $\mathrm{XX}^{\mathrm{e}}$ siècle, Léo-Pol Morin (1892-1941) a largement contribué à l'intégration du répertoire de la modernité sur les scènes musicales du Canada français. Sa solide formation musicale, acquise autant au Québec qu'en France, ainsi que sa plume agile, ont fait de Morin un nom incontournable de la vie musicale montréalaise. Interprète chevronné, ses idées et ses convictions sur la musique de son temps trouvèrent une tribune de choix pendant plus de vingt ans dans des journaux tels que La Patrie, La Presse et Le Canada. Cet article propose un survol des premières années de la participation de Léo-Pol Morin à la vie des concerts ainsi qu'une analyse de ses nombreux écrits portant sur les compositeurs de l'école moderne française, au cœur de laquelle il place Gabriel Fauré, Claude Debussy et Maurice Ravel.

\begin{abstract}
A major figure of Quebec's musical life at the beginning of the 2oth century, Léo-Pol Morin (1892-1941) contributed remarkably to the inclusion of the modern repertoires in the French Canadian concert progammation. Thanks to his strong musical training, in Quebec and France, and to his agile writing style, Morin became a landmark of Montreal's musical life. As well as an experienced musical performer, his ideas and convictions on contemporary music find their way in newspapers such as La Patrie, La Presse, and Le Canada, during a twenty-year period. This essay offers an overview of Léo-Pol Morin's first years of involvement in concert life, and an analysis of his writings addressing the modern French school, and specifically Gabriel Fauré, Claude Debussy and Maurice Ravel.
\end{abstract}

